\title{
Unexpected genomic, biosynthetic and species diversity of Streptomyces bacteria from bats in Arizona and New Mexico, USA
}

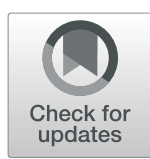

\author{
Cooper J. Park ${ }^{1}$, Nicole A. Caimi ${ }^{2}$, Debbie C. Buecher ${ }^{3}$, Ernest W. Valdez ${ }^{2,4}$, Diana E. Northup ${ }^{2^{*}}$ and \\ Cheryl P. Andam ${ }^{*}$
}

\begin{abstract}
Background: Antibiotic-producing Streptomyces bacteria are ubiquitous in nature, yet most studies of its diversity have focused on free-living strains inhabiting diverse soil environments and those in symbiotic relationship with invertebrates.

Results: We studied the draft genomes of 73 Streptomyces isolates sampled from the skin (wing and tail membranes) and fur surfaces of bats collected in Arizona and New Mexico. We uncovered large genomic variation and biosynthetic potential, even among closely related strains. The isolates, which were initially identified as three distinct species based on sequence variation in the $16 \mathrm{~S}$ rRNA locus, could be distinguished as 41 different species based on genome-wide average nucleotide identity. Of the 32 biosynthetic gene cluster (BGC) classes detected, non-ribosomal peptide synthetases, siderophores, and terpenes were present in all genomes. On average, Streptomyces genomes carried 14 distinct classes of BGCs (range $=9-20$ ). Results also revealed large inter- and intra-species variation in gene content (single nucleotide polymorphisms, accessory genes and singletons) and BGCs, further contributing to the overall genetic diversity present in bat-associated Streptomyces. Finally, we show that genome-wide recombination has partly contributed to the large genomic variation among strains of the same species.

Conclusions: Our study provides an initial genomic assessment of bat-associated Streptomyces that will be critical to prioritizing those strains with the greatest ability to produce novel antibiotics. It also highlights the need to recognize within-species variation as an important factor in genetic manipulation studies, diversity estimates and drug discovery efforts in Streptomyces.
\end{abstract}

Keywords: Streptomyces, Bats, Genome, Average nucleotide identity, Biosynthetic gene clusters

\section{Background}

Many of the drugs used against infectious diseases and other medical disorders have been historically derived from molecules synthesized by environmental microbes, with the most notable belonging to the genus Streptomyces (phylum Actinobacteria) [1-3]. The genotypic and

\footnotetext{
*Correspondence: dnorthup@unm.edu; candam@albany.edu

${ }^{2}$ Department of Biology, University of New Mexico, Albuquerque, NM, USA

${ }^{5}$ Department of Biological Sciences, University at Albany, State University of New York, Albany, NY, USA

Full list of author information is available at the end of the article
}

phenotypic diversity of Streptomyces is remarkably enormous. The current, estimated number of known Streptomyces species is approximately 650 [4], thus making it one of the largest genera in the bacterial domain. Streptomyces are ubiquitous in the environment. They are often found in soil and decaying vegetation $[5,6]$, as well as in extreme environments such as polar regions $[7,8]$, deserts [9], hypersaline sites [10] and the deep sea [11]. Some species form a symbiotic relationship with invertebrates [12], many of which use Streptomyces-produced

(c) The Author(s). 2021 Open Access This article is licensed under a Creative Commons Attribution 4.0 International License, which permits use, sharing, adaptation, distribution and reproduction in any medium or format, as long as you give appropriate credit to the original author(s) and the source, provide a link to the Creative Commons licence, and indicate if changes were made. The images or other third party material in this article are included in the article's Creative Commons licence, unless indicated otherwise in a credit line to the material. If material is not included in the article's Creative Commons licence and your intended use is not permitted by statutory regulation or exceeds the permitted use, you will need to obtain permission directly from the copyright holder. To view a copy of this licence, visit http://creativecommons.org/licenses/by/4.0/. The Creative Commons Public Domain Dedication waiver (http://creativecommons.org/publicdomain/zero/1.0/) applies to the data made available in this article, unless otherwise stated in a credit line to the data. 
antibiotics to protect themselves against infection [12, 13]. For example, beewolf digger wasps cultivate symbiotic Streptomyces species that produce a cocktail of multiple antibiotics for protection against infections [14]. Wasps then deposit a combination prophylaxis of nine different antibiotics into the larval cocoon, a defensive strategy similar to the combination treatment used in human medicine [14]. This results in higher efficacy against a broader spectrum of pathogens and reduces the likelihood of a pathogen developing resistance [14].

The increasing public health burden caused by multidrug resistance and the continuing need to find new treatments against communicable (infectious) and noncommunicable (chronic) diseases suggests that the search for bioactive compounds with novel mechanisms of action or with new cellular targets is greater than ever. Unexplored or rarely visited sites, such as caves that have unique physical and chemical characteristics (e.g., high humidity, low light, limited nutrients), represent a fertile source of antibiotic-producing bacteria for potential use in drug discovery efforts. For example, a genetically diverse assembly of Streptomyces have been identified in various volcanic, limestone and other calcareous caves, including those found on cave walls and in guano [15-18]. When tested against a variety of fungal and bacterial pathogens, some of these Streptomyces exhibited antagonistic activity, thus providing a rich reservoir of pharmaceutically relevant bioactive molecules. Cave-dwelling animals such as bats have also been shown to harbor diverse Streptomyces bacteria, many of which have the ability to inhibit the invasive fungus Pseudogymnoascus destructans [19]. This fungus is the causal agent of white-nose syndrome that affects hibernating bats and has resulted in reduced bat populations in North America [20].

The ability of Streptomyces to successfully inhabit many underexplored or overlooked environments suggests that many novel bioactive compounds remain to be discovered. In this study, we used genomic approaches to explore the diversity of 73 Streptomyces isolates collected from multiple species of bats inhabiting caves in Arizona and New Mexico [19]. Results indicate a remarkably diverse array of Streptomyces species from bats, based on genome-wide average nucleotide identity (ANI) [21]. We also report inter- and intra-species variation in gene content and biosynthetic gene clusters, which further expands the metabolic potential of these bacteria. Our findings provide important insights on bats and caves as unique but poorly studied environmental sources of antibiotic-producing Streptomyces. This knowledge will be critical to addressing the urgent need to discover commercial antibiotics with novel cellular targets or novel molecular activity to inhibit pathogens that threaten the health of humans, bats and other animals.

\section{Results}

Discordance in species boundaries between 16S rRNA gene and genome-wide nucleotide identity

We obtained whole genome sequences of 73 Streptomyces isolates that were sampled from the skin and fur surfaces of healthy bats (i.e., free of white-nose syndrome) that were collected from multiple caves in Arizona and New Mexico, USA (Additional file 1: Table S1). These isolates were selected from a culture collection comprising 632 isolates, which were initially identified using sequence variation in the $16 \mathrm{~S}$ rRNA locus [19]. The 73 isolates came from nine bat species: pallid bat (Antrozous pallidus), Townsend's big-eared bat (Corynorhinus townsendii), big brown bat (Eptesicus fuscus), silverhaired bat (Lasionycteris noctivagans), western smallfooted bat (Myotis ciliolabrum), long-eared bat (Myotis evotis), fringed bat (Myotis thysanodes), cave bat (Myotis velifer) and long-legged bat (Myotis volans). All of these bats are insectivorous, but some have different food preferences depending on when those food items are available. For example, C. townsendii prefers to feed on moths compared to beetles [22], whereas the remaining bats in this study consume a variety of hard-bodied arthropods such as beetles. However, when seasonally abundant and energetically worthwhile, these bats will also feed on moths. Herein, the feeding strategies of the bats include aerial hawking or gleaning of arthropods from different surfaces. Of these bats, only A. pallidus, $M$. evotis and $M$. thysanodes are considered occasional gleaners and are capable of gleaning insects from plants and other substrates [23-25]. Differences in food preferences and wide foraging ranges provide opportunities for bats to come in contact with a variety of microbes, which may partly explain the genetic variation in Streptomyces we observed.

The number of contigs per genome ranged from 69 to 500 and N50 values ranged from 31,031-643,063 bp (Additional file 1: Table S1). We initially used sequence variation in the $16 \mathrm{~S}$ rRNA locus to delineate species boundaries. These isolates can be grouped into three large clusters (Fig. 1a), with each cluster representing a distinct species based on the $97 \%$ sequence similarity threshold in the 16S rRNA gene (Fig. 1b and Additional file 2: Fig. S1). This threshold value has been previously used for taxonomic classification of Streptomyces [4]. Within each of the three clusters, sequence similarities between isolates ranged from 99.41-99.79\%, 96.86$98.47 \%$ and $98.76-99.43 \%$ in clusters 1,2 and 3 , respectively.

Surprisingly, when we used the genome-wide ANI [21] to compare isolates, the majority of isolates within each of the three 16S rRNA-based clusters fell below the 95\% ANI threshold, which is often used to define a species [21] (Fig. 1c and Additional file 3: Table S2). The ANI 


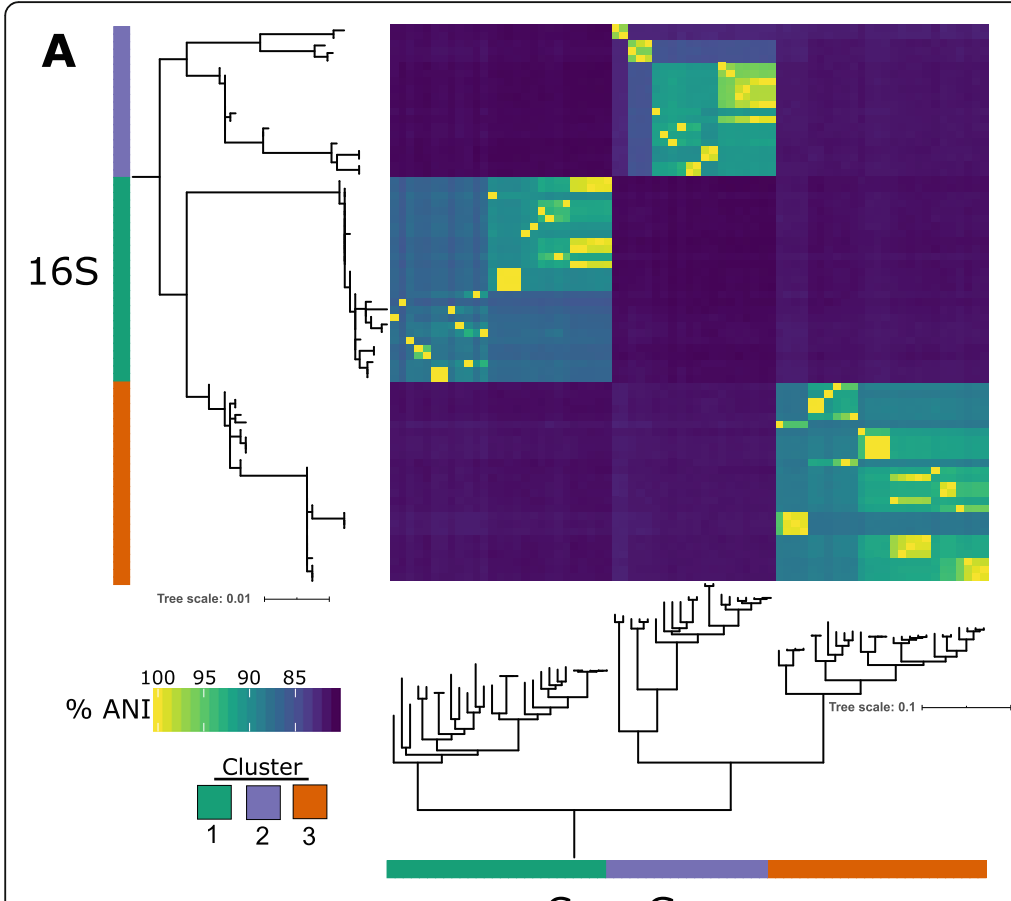

\section{Core Genome}

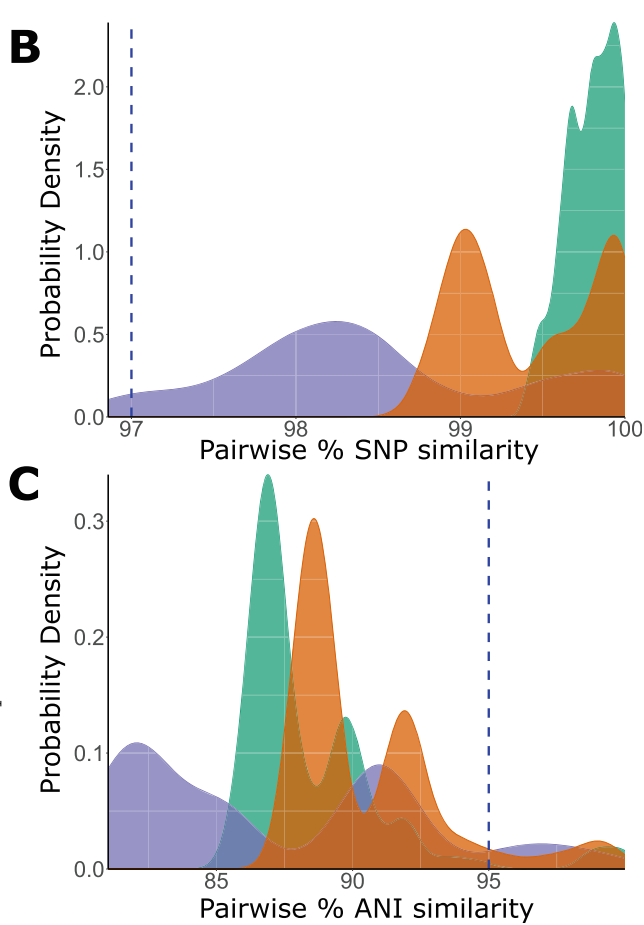

Fig. 1 Phylogenetic and genomic comparison of 73 Streptomyces strains. a Phylogenetic clustering of strains based on 165 rRNA sequence similarity and 1149 core genes. Both trees were midpoint-rooted. Scale bars represent the number of nucleotide substitutions per site. The matrix shows genome-wide, average nucleotide identity (ANI) values calculated for every pair of genomes in the entire data set. Density distribution of all pairwise b 165 rRNA sequence similarity and $\mathbf{c}$ average nucleotide identity (ANI) values. The dashed blue line in each plot indicates the \% threshold often used to define a species (97\% in 16S rRNA and $95 \%$ in ANI). SNP - single nucleotide polymorphisms

metric pertains to the average nucleotide identity of all orthologous genes shared between any two genomes and hence provides a more robust comparison in classifying microbial strains [21]. The clustering into three groups that we observed using $16 \mathrm{~S}$ rRNA sequence variation remained unchanged when we used the 1149 concatenated core genes, which are genes present in $\geq 95 \%$ of the genomes (Additional file 4: Table S3 and Additional file 5: Fig. S2). Within each of the 16S rRNAdefined clusters, genomes exhibited ANI values ranging from $85.53-99.99 \%, 87.72-99.99 \%$, and $81.03-99.99 \%$ in clusters 1, 2 and 3, respectively (Fig. 1a). These results indicate that a species defined by the $16 \mathrm{~S}$ rRNA gene is made up of multiple species based on their genomic sequences. Overall, we can delineate 41 species of Streptomyces using the 95\% ANI threshold in our dataset, indicating a greater level of species diversity than was initially recognized.

\section{Distribution of biosynthetic gene clusters (BGCs)}

Streptomyces are best known for their prolific ability to produce antibiotics and other useful compounds commonly used in human medicine, animal health, industry, and agriculture [1,26]. These compounds are derived from the production of secondary metabolites, which are encoded by a set of physically linked genes called BGCs $[3,27]$. The genes in a BGC function in peptide assembly, regulation, resistance and synthesis of a secondary metabolite [28, 29]. Secondary metabolites are compounds that are not required for growth but may confer a certain advantage to their producers in a given environment. A previous study of bat-associated Streptomyces reported potent antagonistic activity against the fungal pathogen $P$. destructans [19]. Hence, we hypothesized that Streptomyces bacteria from southwestern bat species harbor an abundant and diverse suite of BGCs.

From our analyses, we detected a total of 32 major classes of BGCs (excluding BGCs identified as others and fused; Fig. 2 and Additional file 6: Table S4), which is consistent with previous studies of Streptomyces from other environmental sources [6, 27, 30]. On average, a genome carried 14 distinct classes of BGCs (range $=9-20$ ). Of the 32 BGC classes detected, we found non-ribosomal peptide synthetases (NRPS), siderophores, and terpenes present in all genomes. Other BGCs that were commonly found in Streptomyces included bacteriocin (present in 72 genomes), type 1 polyketide synthase (t1PKS; 72 genomes), butyrolactone (71 genomes) and type 3 PKS (t3PKS; 70 genomes). In contrast, some BGCs were present in only a handful of genomes. These included oligosaccharide $(8$ 


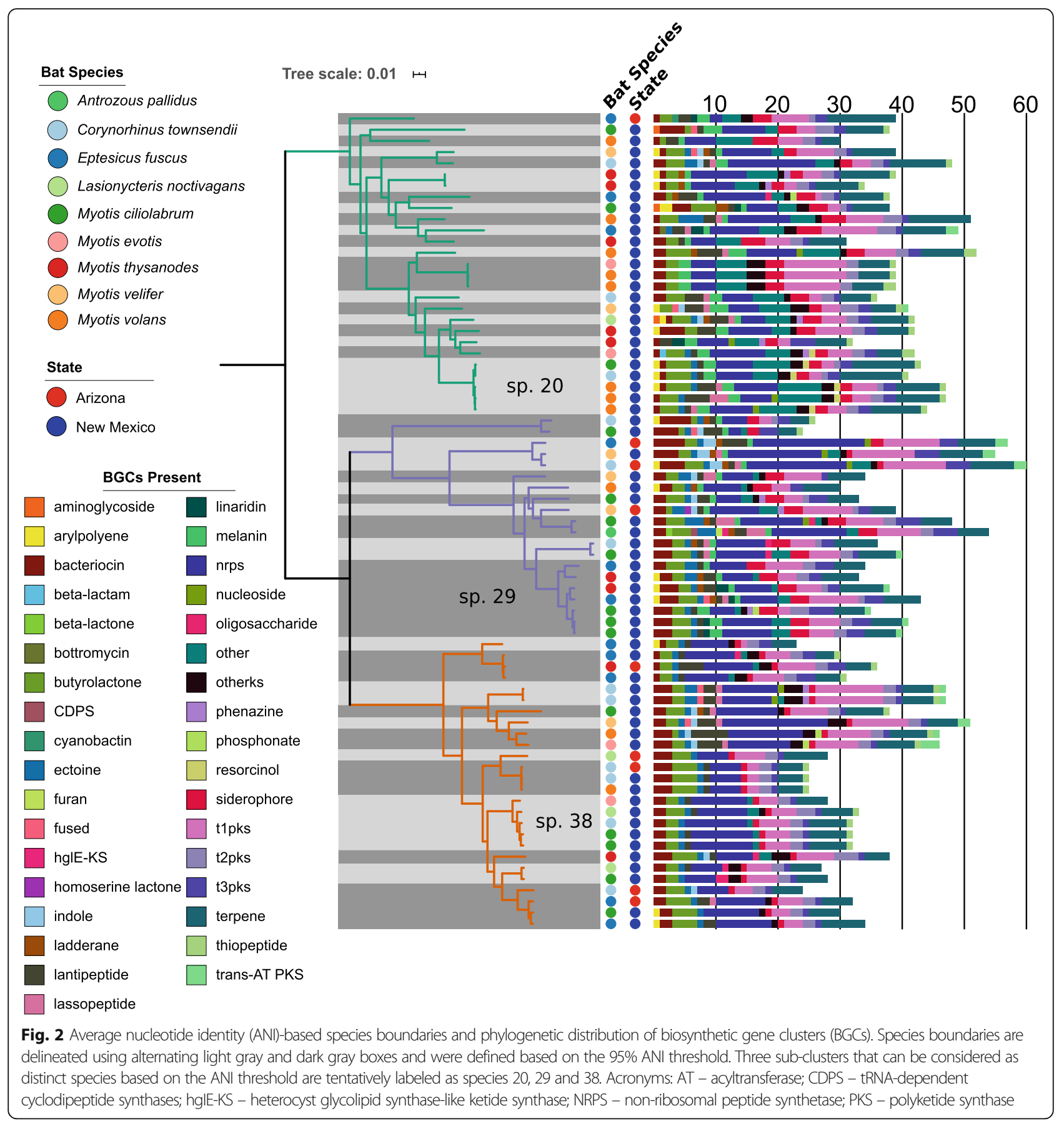

genomes), linaridin (8 genomes), resorcinol (6 genomes), phosphonate (4 genomes), aminoglycoside (3 genomes), bottromycin (1genome), cyanobactin (1 genome), betalactam (1 genome) and homoserine lactone (1 genome). We also note that a genome may harbor multiple copies of a BGC class. For example, the number of NRPS in a single genome ranged from 2 to 18 (mean $=7.5)$. The number of siderophore copies in a single genome ranged from 1 to 4 (mean $=2.1)$. The number of terpene copies in a single genome ranged from 3 to $14($ mean $=5.9)$. We did not identify any specific class of BGC that is unique to any of the three ANI clusters nor to any of the nine bat species.

Genome variation between strains in three select species We wanted to further investigate the extent of genomic variation among strains within a species. There were three sub-clusters that can be considered as distinct species based on the ANI threshold and that consists of multiple strains. These are tentatively labeled as species 
20, 29 and 38 in Fig. 2. These three subclusters each had five, seven and five genomes, respectively (Fig. 3a). We first estimated the pan-genome of each species, which consisted of 13,461, 20,342 and 20,474 genes in species 20, 29 and 38, respectively (Fig. 3b and Additional file 7: Table S5). The total number of core genes were 6591, 5125 and 5979, while the total number of accessory genes were $6870,15,217$ and 14,495 for species 20,29 and 38 , respectively.

We found differences in the number and classes of BGCs, including hybrid BGCs (i.e., BGCs with genes that code for more than one type of scaffold-synthesizing enzymes [28, 31]), between strains of the same species (Fig. 3c). Some major classes of BGCs were present in all genomes across all three species, such as bacteriocin, butyrolactone, ectoine, NRPS, siderophore, types 1, II and III PKS and terpene. However, we also identified BGCs that were strain-specific (lassopeptide, phenazine, and phosphonate in species 29) and species-specific (nucleoside and resorcinol in species 20, and ladderane, linaridin, phenazine, phosphonate and transATPKS in species 29). If we further classify the hybrid BGCs based on their individual BGC components, we can further observe greater diversity among strains. For example, we found species-specific cases of butyrolactone-arylpolyeneNRPS (species 20), butyrolactone-other KS (species 20), melanin-ladderane-arylpolyene-NRPS (species 29), melaninNRPS (species 29), t1PKS-butyrolactone-NRPS (species 29), t1PKS-other KS (species 20), t2PKS-butyrolactone, (species 38) t2PKS-terpene (species 38), t3PKS-t1PKS, (species 29) t3PKS-terpene (species 20), and terpene-linaridin (species 29). We also found strain-specific presence of t1PKSlinaridin, t2PKS-otherKS and t2PKS-t3PKS-otherKSphenazine.

The total number of protein coding genes per genome ranged from 8666-9364, 8932-9974, 8071-8513 in species 20, 29 and 38, respectively. Species 38 had significantly fewer genes than either species 20 or species 29 (Fig. 3d) (Mann-Whitney U pairwise test). However, we did not find any significant differences in the number of either accessory genes (Fig. 3e) or singleton genes (i.e., genes present only in a single genome) (Fig. 3f) between species. These results indicate that inter-strain variation

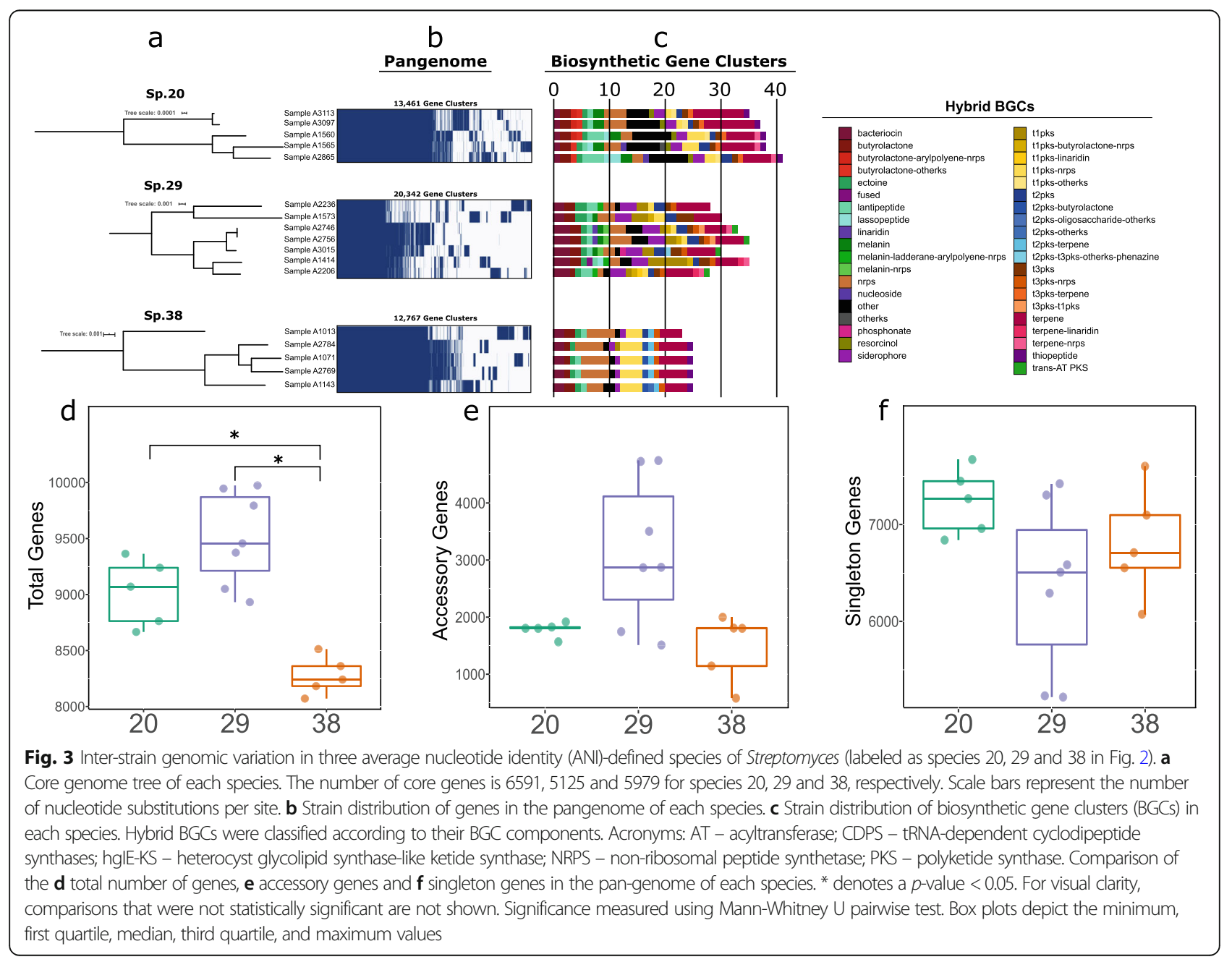


in gene content further contributes to the overall genetic diversity present in bat-associated Streptomyces.

\section{Recombination within a species}

Streptomyces are known to frequently recombine [3234 ] which may partly explain the observed genomic variation between strains within each of species 20, 29 and 38 . Using the core genome alignment of each of the three species, we tested for evidence of recombination using the.

Pairwise Homoplasy Index test and Splitstree network analysis. The Splitstree analysis shows the reticulations in the phylogenetic relationships between strains of each species (Fig. 4). The networks also reveal that differences in host species do not appear to hinder recombination between strains. However, only species 20 and 38 show significant signal for recombination $(p$-value $=0.0)$, while species 29 does not $(p$-value $=1.0)$.

\section{Discussion}

Antibiotic-producing Streptomyces bacteria are ubiquitous in nature, yet most studies of its diversity have focused on those free-living strains inhabiting diverse soil environments $[5,6]$ and those in symbiotic relationship with invertebrates (e.g., insects, marine sponges) [14, 35, 36]. Most commercially used Streptomyces-derived antibiotics today, such as streptomycin [37], were originally derived from strains collected from soils. However, investigations on the prevalence, diversity and contributions of Streptomyces to their vertebrate animal hosts remain limited. A previous study reported the remarkable species diversity of $20 \%$ of a large culture collection of bat-associated Streptomyces at the University of New

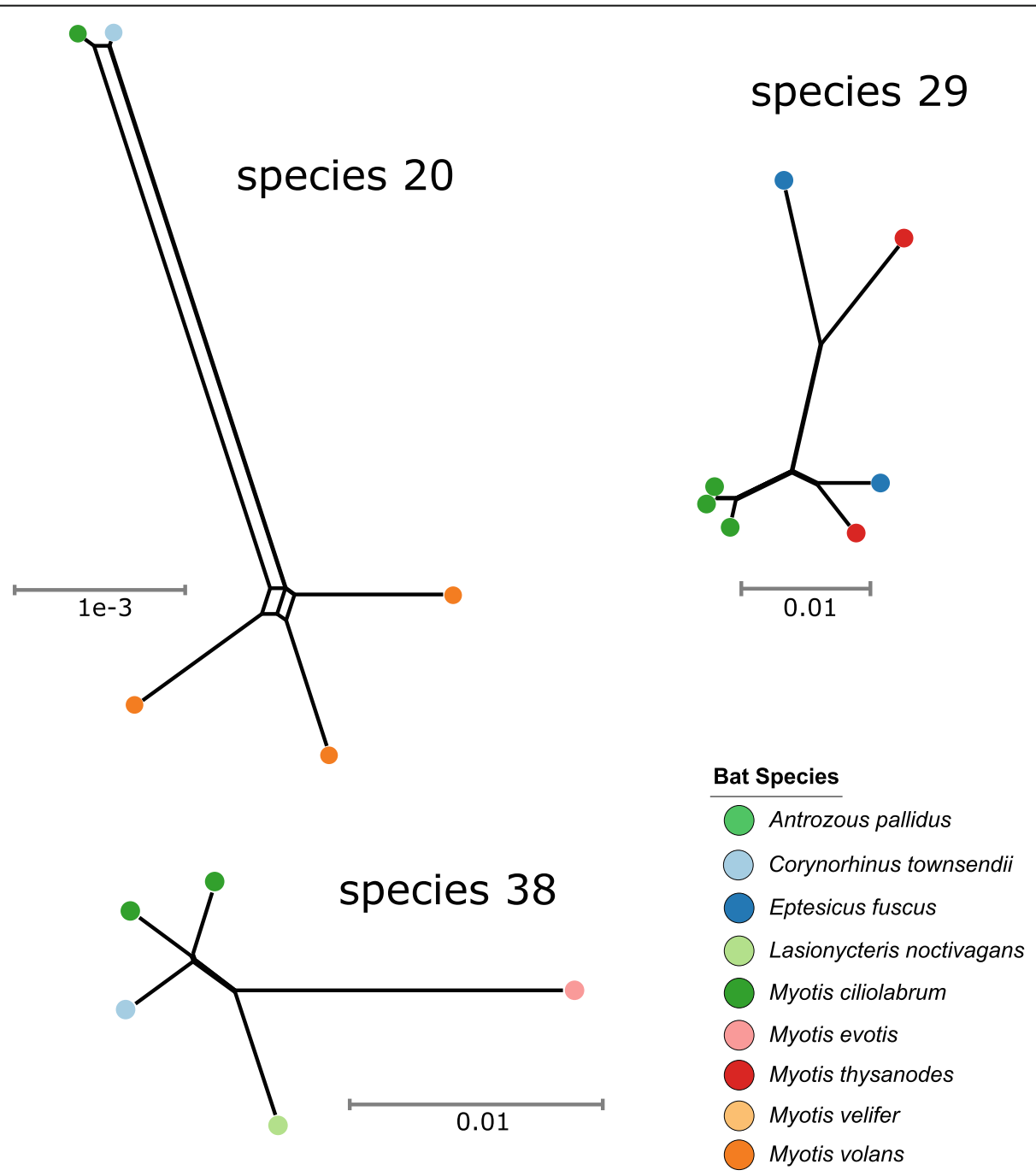

Fig. 4 A phylogenetic network of the core genome of Streptomyces species 20, 29 and 38 generated using SplitsTree4. The dots represent the host bat species and colors are identical to those in Fig. 2 
Mexico (UNM) [19]. In that study, sequence variation in the 16S rRNA and five other housekeeping genes were used to characterize the phylogenetic diversity and relationships of Streptomyces, with isolates representing 15 novel species that exhibited antifungal activity [19]. Using 73 Streptomyces isolates from the UNM culture collection, we uncovered large genomic variation and biosynthetic potential, even among closely related strains. Bats are therefore an important yet underappreciated source of antibiotic-producing microbes. Our study provides an initial genomic assessment of batassociated Streptomyces that will be critical to prioritizing those strains with the greatest ability to produce novel anti-fungal compounds.

It has long been recognized that species classification in Streptomyces is problematic, being driven historically more by the type of antibiotic produced and patent issues rather than by genetic, ecological, or evolutionary data [38]. Recent studies on different Streptomyces species defined by the $16 \mathrm{~S}$ rRNA locus revealed a striking illustration of how traditional species classification can complicate drug discovery schemes or overlook singular bacterial strains. For example, two strains of Streptomyces griseus from geographically disparate locations but with identical 16S rRNA sequences, exhibited differences in spore pigmentation, amount of spore formation, aerial hyphae distribution on the colony, and production of secondary metabolites [39]. These differences may be partly explained by adaptive processes to specific environmental conditions. In another study, ten Streptomyces strains from different lichen species but with 16S rRNA gene sequences identical to the type strain Streptomyces cyaneofuscatus JCM 4364 exhibited highly variable phenotypic (colony morphology and color, halotolerance, optimal $\mathrm{pH}$ growth), metabolic and genomic features, such that they could be distinguished as five different species [40].

The large amount of genomic and BGC variation between genomes, even between closely related isolates, may reflect fine-scale differences in their adaptive potential. For example, the presence on all genomes of siderophores, which are involved in the acquisition of ferric ions, may reflect the need for Streptomyces to cope in environments with limited iron supply [41]. However, differences in the abundance of siderophores per genome may be due to interactions with specific bacterial partners, as has been previously reported $[42,43]$. Future work should focus on investigating the functional role of structurally distinct types of siderophores as well as other BGCs in Streptomyces' adaptation to different bat species that harbor them and to the cave environment.

Our study presents several limitations that need to be recognized. First, only culturable isolates were used in this study. Cultivation techniques, while effective in isolating individuals from a microbial community, can unintentionally bias findings on community composition and functions. This is because cultivation fails to discover novel strains that are recalcitrant to specific cultivation methods, media and laboratory conditions commonly used because they do not precisely mimic the ecological niche of the bacterium in nature. Hence, we recognize that the extent of genomic diversity we found in our dataset is likely an under-estimation of the true scale of Streptomyces diversity in bats, suggesting that additional species with unique genetic and phenotypic features remain to be discovered. This will also hold true for exploring BGC diversity. Second, we did not sample Streptomyces from the cave walls, where bats may pick up Streptomyces spores and hence influence their genetic diversity. Another limitation of our study is the assembly quality of our genomes. Illumina sequencing of large bacterial chromosomes remains a challenge due to chimeric sequences and sequencer errors. Furthermore, genome regions with high $\mathrm{G}+\mathrm{C}$ content [such as Streptomyces, which typically has over $70 \% \mathrm{G}+\mathrm{C}$ content [44]] can be sequenced with lower coverage than the rest of the genome due to biases in the amplification step [45]. These challenges can exacerbate the amount of gene content variation detected in genomes. In this study, the assembly quality of our genomes could take the form of erroneously large accessory genomes and/or improperly characterized BGC content. Future studies should utilize long-read sequencing technologies alongside short-reads to generate more complete hybrid assemblies which can overcome these common sequencing challenges [46].

\section{Conclusions}

Overall, our study provides an initial genome-based assessment of the bat-associated Streptomyces diversity that will be critical to prioritizing those strains with the greatest ability to produce novel bioactive compounds, including those that can strongly inhibit $P$. destructans and other mycotic diseases. We emphasize the value of poorly explored settings, such as caves and bats, as important resources of antibiotic-producing bacteria for current drug discovery efforts relevant to human and veterinary medicine.

\section{Methods \\ Collection and isolation of Streptomyces}

Isolates in our study came from a culture collection of which a subset was used in a previously published dataset of Streptomyces sampled from the skin (wing and tail membranes) and fur surfaces of bats [19]. Details on bat collection protocols, sampling permits and bacterial isolation procedures were described in reference [19]. Bats were caught using mist nets or were hand plucked from 
cave walls, according to approved protocols under the following collection permits: 2014 Arizona and New Mexico Game and Fish Department Scientific Collecting Permit (SP670210, SCI\#3423, and SCI\#3350), National Park Service Scientific Collecting Permit (CAVE-2014-SCI-0012, CAVE-2015-SCI-0011 ELMA-2013-SCI-0005, ELMA2014-SCI-0001, CHIR-2015-SCI-0001, and PARA-2012SCI-0003), U.S. Geological Survey Fort Collins Science Center Standard Operating Procedure (SOP) 2013-01, and an Institutional Animal Care and Use Committee (IACUC) permit from the University of New Mexico (protocol \#12-100835-MCC and Protocol 15101307MC) and from the National Park Service (protocol \#IMRELMA.PARA Northup-Bat-2013.A2 and NPS Protocol Number IMR_ELMA.PARA.CAVE.SEAZ_Northup_Bats_ 2015.A2). All experimental protocols were approved by the institutions and licensing committees listed above. Bats were swabbed from caves post-hibernation or from netting on the surface near drinking sources. Sampling was carried out in 2013-2015. Four actinobacteriumselective media were used to isolate Streptomyces (Actinomycete isolation agar [Difco, Sparks, Maryland], gellan gum agar, humic acid-vitamin agar and glucose yeast extract agar) supplemented with cycloheximide, nalidixic acid, trimethoprim and a vitamin solution. Immediately following swabbing of each bat, plates were inoculated and kept at $4{ }^{\circ} \mathrm{C}$ during transport and at $20^{\circ} \mathrm{C}$ in the laboratory during initial growth. Initial Streptomyces identification was done by extracting and sequencing the $16 \mathrm{~S}$ rRNA locus using Sanger sequencing [19].

\section{DNA extraction and whole genome sequencing}

Pure cultures were grown in R2B broth medium (Difco, Sparks, Maryland) at $37^{\circ} \mathrm{C}$ for $24-72 \mathrm{~h}$. DNA was extracted and purified from cultures using the DNeasy Extraction kit (Qiagen, Germantown, Maryland) following manufacturer's protocols. DNA concentration and quality were measured using a Nanodrop spectrophotometer and Qubit 4 fluorometer. DNA libraries were prepared using the NexteraXT protocol (as per the manufacturer's instructions) with $1 \mathrm{ng}$ of genomic DNA per strain. Samples were sequenced as multiplexed libraries on the Illumina HiSeq platform operated per the manufacturer's instructions to produce paired end reads of 250 nucleotides in length. Sequencing was done at the University of New Hampshire Hubbard Center for Genome Studies, Durham, New Hampshire, USA.

\section{Genome assembly and annotation}

Reads were assembled into contigs using the de novo assembler SPAdes v.3.13.1 that was developed specifically for bacterial genomes [47]. Genome assembly quality was assessed using QUAST [48]. We also selected only those genomes with $<500$ contigs. In total, we used 73 draft genomes for all downstream analyses. The resulting contigs in each genome were annotated using Prokka, a stand-alone tool that combines multiple feature prediction tools to identify coding sequences, ribosomal and transfer RNA genes, non-coding RNA and signal leader peptides in bacterial genomes [49].

\section{Pan-genome and phylogenetic analysis}

To determine the degree of genomic relatedness and hence clarify the taxonomic breadth within our dataset, we calculated the genome-wide ANI for all possible pairs of genomes using the program FastANI v.1.0 [21]. We used the program Roary [50] to characterize the core and accessory genes in the pan-genome of the 73 strains. However, Roary's default parameters assume a species level relationship among genomes. To account for the greater genomic variation in our genus-scale dataset, we used the mean pairwise fastANI value for the entire genus $(81 \%)$ as the minimum percent identity between orthologous genes (parameter '-i 81). For the speciesspecific Roary analyses, we used the standard $95 \%$ that is commonly used to define species boundaries [50]. Significance in gene content between clusters was measured using Mann-Whitney U pairwise test.

We aligned the sequences of the 16S rRNA gene extracted from the genomes using MAFFT [51] and counted all pairwise single nucleotide polymorphism (SNP) differences using snp-dists v0.6.3 (https://github. com/tseemann/snp-dists). A $16 \mathrm{~S}$ rRNA phylogenetic tree was built using RAxML v.8.2.11 [52] with a general-time reversible (GTR) nucleotide substitution model [53], four gamma categories for rate heterogeneity and 100 bootstrap replicates. We also built a phylogenetic tree using the concatenated sequence alignments of the core genes using RAxML with the GTR model and four gamma categories. All trees were visualized using the Interactive Tree of Life [54].

BGCs encoding secondary metabolites were predicted and annotated using the standalone version of antiSMASH v.4.1 with default parameters, which identifies BGCs using a signature profile Hidden Markov Model based on multiple sequence alignments of experimentally characterized signature proteins or protein domains [55]. Due to their high number, hybrid BGCs were split and counted as individual BGC classes in the genus tree (e.g., terpene-t1PKS BGC would count as both one terpene and one t1PKS). In species-level comparisons, we showed all unique hybrid BGCs.

\section{Recombination analysis}

Using the alignment of the concatenated core genes of each of the three species as input, we ran the Pairwise Homoplasy Index test for recombination with 100 permutations using PhiPack [56]. The PHI test calculates a 
pairwise incompatibility score of each nucleotide site in an alignment. The $p$-value for the PHI test was calculated under the null hypothesis of no recombination. Recombinations were visualized using SplitsTree v4.14.4 which integrates reticulations due to recombinations in phylogenetic relationships [57].

All methods were carried out in accordance with relevant guidelines and regulations at the National Park Service, Arizona and New Mexico Game and Fish Departments, U.S. Geological Survey, University of New Mexico and University at Albany.

\section{Supplementary Information}

The online version contains supplementary material available at https://doi. org/10.1186/s12864-021-07546-w.

Additional file 1: Table S1. Accession numbers, metadata and genome characteristics of the 73 Streptomyces isolates. CDS - coding sequence.

Additional file 2: Fig. S1. $16 \mathrm{~S}$ rRNA tree of Streptomyces isolates with bootstrap values.

Additional file 3: Table S2. Average nucleotide identity (ANI) values for all pairs of genomes of Streptomyces isolates calculated using fastANI.

Additional file 4: Table S3. Pan-genome analysis of the 73 Streptomyces genomes estimated using Roary.

Additional file 5: Fig. S2. Core genome tree generated from 1149 concatenated core genes of Streptomyces identified by Roary.

Additional file 6: Table S4. Biosynthetic gene clusters (BGCs) of all genomes of Streptomyces estimated using antiSMASH.

Additional file 7: Table S5. Pan-genome analyses of Streptomyces sp. 20, 29 and 38 in Fig. 2 estimated using Roary.

\section{Acknowledgements}

We thank the staff at El Malpais and Grand Canyon-Parashant National Monuments, El Malpais National Conservation Area, Carlsbad Caverns National Park, the Southeast Arizona Group of National Park Sites, the Bureau of Land Management and the Fort Stanton Cave Study Project. We also thank the students in the Northup lab at the University of New Mexico for assisting with our bat netting efforts.

The authors thank the University of New Hampshire Resource Computing Center where all bioinformatics analyses were performed.

\section{Authors' contributions}

C.J.P. carried out DNA library preparation for sequencing and all bioinformatics analyses. N.A.C carried out bacterial culturing and isolation. E.W.V., D.E.N. and D.C.B. carried out bat identification and sample collection. C.P.A., D.E.N., C.J.P., and E.W.V. wrote the manuscript. All authors read and approved the final manuscript

\section{Funding}

Funding for the creation of the bat Actinobacteria culture collection in the Northup lab at UNM was provided by the Eppley Foundation, the National Park Service through the Colorado Plateau Cooperative Ecosystem Studies unit (CPCESU) for work at the El Malpais National Monument, Carlsbad Caverns National Monument, and Grand Canyon-Parashant National Monument. The Western National Park Association provided funding for the El Malpais National Monument work. The Bureau of Land Management and Fort Stanton Cave Study Project funded work in BLM Caves 45 and 55 and Fort Stanton Cave, respectively. Additional funding was provided by the New Mexico Game and Fish Department Share with Wildlife Program, Cave Conservancy Foundation, National Speleological Society Rapid Response Fund, and T\&E, Inc. This material is based upon work supported by the National Science Foundation (grant number 2055120) and start-up funds from the UAlbany College of Arts and Sciences to C.P.A. The funders had no role in the study design, data collection and interpretation, or the decision to submit the work for publication. Any use of trade, firm, or product names is for descriptive purposes only and does not imply endorsement by the U.S. Government.

\section{Availability of data and materials}

Genome sequence data of the 73 isolates have been deposited in the NCB Sequence Read Archive under BioProject ID PRJNA673820 with BioSample accession numbers listed in Additional file 1: Table S1. The raw data in the NCBI database serve as an acceptable repository by U.S. Geological Survey's standards.

\section{Declarations}

Ethics approval and consent to participate Not applicable.

Consent for publication

Not applicable.

\section{Competing interests}

The authors declare that they have no competing interests.

\section{Author details}

'Department of Molecular, Cellular and Biomedical Sciences, University of New Hampshire, Durham, NH, USA. ²Department of Biology, University of New Mexico, Albuquerque, NM, USA. ${ }^{3}$ Buecher Biological Consulting, Tucson, AZ, USA. ${ }^{4}$ U.S. Geological Survey, Fort Collins Science Center, Fort Collins, CO, USA. ${ }^{5}$ Department of Biological Sciences, University at Albany, State University of New York, Albany, NY, USA.

Received: 15 January 2021 Accepted: 19 March 2021

Published online: 07 April 2021

\section{References}

1. Procópio RE d L, Silva IR d, Martins MK, Azevedo JL d, Araújo JM d. Antibiotics produced by Streptomyces. Braz J Infect Dis. 2012;16:466-71.

2. van der Meij A, Worsley SF, Hutchings MI, van Wezel GP. Chemical ecology of antibiotic production by actinomycetes. FEMS Microbiol Rev. 2017;41(3): 392-416. https://doi.org/10.1093/femsre/fux005.

3. van Bergeijk DA, Terlouw BR, Medema MH, van Wezel GP. Ecology and genomics of Actinobacteria: new concepts for natural product discovery. Nat Rev Microbiol. 2020:18(10):546-58.

4. Labeda DP, Goodfellow M, Brown R, Ward AC, Lanoot B, Vanncanneyt M, et al. Phylogenetic study of the species within the family Streptomycetaceae. Antonie Van Leeuwenhoek. 2012;101(1):73-104. https:// doi.org/10.1007/s10482-011-9656-0.

5. Laskaris P, Tolba S, Calvo-Bado L, Wellington EM, Wellington L. Coevolution of antibiotic production and counter-resistance in soil bacteria. Environ Microbiol. 2010;12(3):783-96. https:/doi.org/10.1111/j.1462-2920.2009.02125.x.

6. Nicault M, Tidjani A-R, Gauthier A, Dumarcay S, Gelhaye E, Bontemps C, et al. Mining the biosynthetic potential for specialized metabolism of a Streptomyces soil community. Antibiotics (Basel). 2020;9(5):271.

7. Augustine N, Wilson PA, Kerkar S, Thomas S. Arctic actinomycetes as potential inhibitors of Vibrio cholerae biofilm. Curr Microbiol. 2012;64(4): 338-42. https://doi.org/10.1007/s00284-011-0073-4.

8. Encheva-Malinova M, Stoyanova M, Avramova H, Pavlova Y, Gocheva B, Ivanova I, et al. Antibacterial potential of streptomycete strains from Antarctic soils. Biotechnol Biotechnol Equip. 2014;28(4):721-7. https://doi. org/10.1080/13102818.2014.947066

9. Arocha-Garza HF, Canales-Del Castillo R, Eguiarte LE, Souza V, De la TorreZavala S. High diversity and suggested endemicity of culturable Actinobacteria in an extremely oligotrophic desert oasis. PeerJ. 2017;5: e3247. https://doi.org/10.7717/peerj.3247.

10. Zhao F, Qin Y-H, Zheng X, Zhao H-W, Chai D-Y, Li W, et al. Biogeography and adaptive evolution of Streptomyces strains from saline environments. Sci Rep. 2016:6(1):32718. https://doi.org/10.1038/srep32718.

11. Sarmiento-Vizcaíno A, González V, Braña AF, Palacios JJ, Otero L, Fernández $J$, et al. Pharmacological potential of phylogenetically diverse actinobacteria isolated from Deep-Sea coral ecosystems of the submarine Avilés canyon in the Cantabrian Sea. Microb Ecol. 2017:73(2):338-52. https://doi.org/10.1007/ s00248-016-0845-2. 
12. Seipke RF, Kaltenpoth M, Hutchings MI. Streptomyces as symbionts: an emerging and widespread theme? FEMS Microbiol Rev. 2012;36(4):862-76. https://doi.org/10.1111/j.1574-6976.2011.00313.x.

13. Kaltenpoth M, Göttler W, Herzner G, Strohm E. Symbiotic bacteria protect wasp larvae from fungal infestation. Curr Biol. 2005;15(5):475-9. https://doi org/10.1016/j.cub.2004.12.084.

14. Kroiss J, Kaltenpoth M, Schneider B, Schwinger M-G, Hertweck C, Maddula RK, et al. Symbiotic Streptomycetes provide antibiotic combination prophylaxis for wasp offspring. Nat Chem Biol. 2010;6(4):261-3. https://doi. org/10.1038/nchembio.331.

15. Riquelme C, Marshall Hathaway JJ, Enes Dapkevicius M d LN, Miller AZ, Kooser A, Northup DE, et al. Actinobacterial diversity in volcanic caves and associated geomicrobiological interactions. Front Microbiol. 2015;6:1342.

16. Axenov-Gribanov DV, Axenov-Gibanov DV, Voytsekhovskaya IV, Tokovenko BT, Protasov ES, Gamaiunov SV, et al. Actinobacteria isolated from an underground lake and moonmilk speleothem from the biggest conglomeratic karstic cave in Siberia as sources of novel biologically active compounds. PLoS One. 2016;11(2):e0149216. https://doi.org/10.1371/journal. pone.0149216.

17. Adam D, Maciejewska M, Naômé A, Martinet L, Coppieters W, Karim L, et al. Isolation, characterization, and antibacterial activity of hard-to-culture Actinobacteria from cave moonmilk deposits. Antibiotics (Basel). 2018;7(2):28.

18. Wiseschart A, Mhuantong W, Tangphatsornruang S, Chantasingh D, Pootanakit K. Shotgun metagenomic sequencing from Manao-Pee cave, Thailand, reveals insight into the microbial community structure and its metabolic potential. BMC Microbiol. 2019;19(1):144. https://doi.org/10.1186/ s12866-019-1521-8

19. Hamm PS, Caimi NA, Northup DE, Valdez EW, Buecher DC, Dunlap CA, et al. Western bats as a reservoir of novel Streptomyces species with antifungal activity. Appl Environ Microbiol. 2017;83(5). https:/doi.org/10.1128/AEM.03057-16.

20. Drees KP, Lorch JM, Puechmaille SJ, Parise KL, Wibbelt G, Hoyt JR, et al. Phylogenetics of a fungal invasion: origins and widespread dispersal of white-nose syndrome. mBio. 2017;8(6):e01941-17. https://doi.org/10.1128/ mBio.01941-17.

21. Jain C, Rodriguez-R LM, Phillippy AM, Konstantinidis KT, Aluru S. High throughput ANI analysis of 90K prokaryotic genomes reveals clear species boundaries. Nat Commun. 2018;9(1):5114. https://doi.org/10.1038/s41467-01 8-07641-9.

22. Kunz TH, Martin RA. Plecotus townsendii. Mamm Species. 1982;175:1-6.

23. O'Farrell MJ, Studier EH. Myotis thysanodes. Mammalian species; 1980. p. 1-5.

24. Manning RW, Jones JK. Myotis evotis. Mamm Species. 1989;329:1-5.

25. Ammerman LK, Hice CL, Schmidley DJ. Bats of Texas. College Station: Texas A\&M University Press; 2012

26. Běhal V. Bioactive products from Streptomyces. Adv Appl Microbiol. 2000;47: 113-56. https://doi.org/10.1016/S0065-2164(00)47003-6.

27. Doroghazi JR, Metcalf WW. Comparative genomics of actinomycetes with a focus on natural product biosynthetic genes. BMC Genomics. 2013;14(1):611. https://doi.org/10.1186/1471-2164-14-611.

28. Cimermancic P, Medema MH, Claesen J, Kurita K, Wieland Brown LC, Mavrommatis $\mathrm{K}$, et al. Insights into secondary metabolism from a global analysis of prokaryotic biosynthetic gene clusters. Cell. 2014;158(2):412-21. https://doi.org/10.1016/j.cell.2014.06.034.

29. Medema $\mathrm{MH}$, Kottmann $\mathrm{R}$, Yilmaz $\mathrm{P}$, Cummings $\mathrm{M}$, Biggins J B, Blin $\mathrm{K}$, et al. Minimum information about a biosynthetic gene cluster. Nat Chem Biol. 2015;11(9):625-31. https://doi.org/10.1038/nchembio.1890.

30. Belknap KC, Park CJ, Barth BM, Andam CP. Genome mining of biosynthetic and chemotherapeutic gene clusters in Streptomyces bacteria. Sci Rep. 2020;10(1):2003. https://doi.org/10.1038/s41598-020-58904-9.

31. Zotchev SB. Genomics-based insights into the evolution of secondary metabolite biosynthesis in actinomycete bacteria. In: Pontarotti P, editor. Evolutionary biology: genome evolution, speciation, coevolution and origin of life. Cham: Springer International Publishing; 2014. p. 35-45. https://doi. org/10.1007/978-3-319-07623-2_2.

32. Doroghazi JR, Buckley DH. Widespread homologous recombination within and between Streptomyces species. ISME J. 2010;4(9):1136-43. https://doi. org/10.1038/ismej.2010.45.

33. Park CJ, Andam CP. Within-species genomic variation and variable patterns of recombination in the tetracycline producer Streptomyces rimosus. Front Microbiol. 2019;10:552. https://doi.org/10.3389/fmicb.2019.00552.

34. Tidjani A-R, Lorenzi J-N, Toussaint $M$, van Dijk E, Naquin D, Lespinet $O$, et al. Massive gene flux drives genome diversity between sympatric
Streptomyces conspecifics. mBio. 2019;10(5):e01533-19. https://doi.org/1 0.1128/mBio.01533-19.

35. Scott JJ, Oh D-C, Yuceer MC, Klepzig KD, Clardy J, Currie CR. Bacterial protection of beetle-fungus mutualism. Science. 2008;322(5898):63. https:// doi.org/10.1126/science.1160423.

36. Almeida EL, Carrillo Rincón AF, Jackson SA, Dobson ADW. Comparative genomics of marine sponge-derived Streptomyces spp. isolates SM17 and SM18 with their closest terrestrial relatives provides novel insights into environmental niche adaptations and secondary metabolite biosynthesis potential. Front Microbiol. 2019;10:1713.

37. Waksman SA. Streptomycin: background, isolation, properties, and utilization. Science. 1953;118(3062):259-66. https://doi.org/10.1126/ science.118.3062.259.

38. Anderson AS, Wellington EM. The taxonomy of Streptomyces and related genera. Int J Syst Evol Microbiol. 2001;51(Pt 3):797-814. https://doi.org/10.1 099/00207713-51-3-797.

39. Sottorff I, Wiese J, Lipfert M, Preußke N, Sönnichsen FD, Imhoff JF. Different secondary metabolite profiles of phylogenetically almost identical Streptomyces griseus strains originating from geographically remote locations. Microorganisms. 2019;7(6). https://doi.org/10.3390/microorga nisms7060166.

40. Antony-Babu S, Stien D, Eparvier V, Parrot D, Tomasi S, Suzuki MT. Multiple Streptomyces species with distinct secondary metabolomes have identical 16S rRNA gene sequences. Sci Rep. 2017;7(1):11089. https://doi.org/10.1038/ s41598-017-11363-1.

41. Eto D, Watanabe K, Saeki H, Oinuma K, Otani K, Nobukuni M, et al. Divergent effects of desferrioxamine on bacterial growth and characteristics. J Antibiot (Tokyo). 2013;66(4):199-203. https://doi.org/10.1038/ja.2012.111.

42. Traxler MF, Watrous JD, Alexandrov T, Dorrestein PC, Kolter R. Interspecies interactions stimulate diversification of the Streptomyces coelicolor secreted metabolome. mBio. 2013;4(4):e00459-13.

43. Kramer J, Özkaya Ö, Kümmerli R. Bacterial siderophores in community and host interactions. Nat Rev Microbiol. 2020;18(3):152-63. https://doi.org/10.1 038/s41579-019-0284-4.

44. Barka EA, Vatsa P, Sanchez L, Gaveau-Vaillant N, Jacquard C, Klenk H-P, et al. Taxonomy, physiology, and natural products of Actinobacteria. Microbiol Mol Biol Rev. 2015;80:1-43.

45. Aird D, Ross MG, Chen W-S, Danielsson M, Fennell T, Russ C, et al. Analyzing and minimizing PCR amplification bias in Illumina sequencing libraries. Genome Biol. 2011;12(2):R18. https://doi.org/10.1186/gb-2011-12-2-r18.

46. Lee N, Kim W, Hwang S, Lee Y, Cho S, Palsson B, et al. Thirty complete Streptomyces genome sequences for mining novel secondary metabolite biosynthetic gene clusters. Sci Data. 2020;7(1):55. https://doi.org/10.1038/s41 597-020-0395-9.

47. Bankevich A, Nurk S, Antipov D, Gurevich AA, Dvorkin M, Kulikov AS, et al. SPAdes: a new genome assembly algorithm and its applications to singlecell sequencing. J Comput Biol. 2012;19(5):455-77. https://doi.org/10.1089/ cmb.2012.0021.

48. Gurevich A, Saveliev V, Vyahhi N, Tesler G. QUAST: quality assessment tool for genome assemblies. Bioinformatics. 2013;29(8):1072-5. https://doi.org/1 0.1093/bioinformatics/btt086

49. Seemann T. Prokka: rapid prokaryotic genome annotation. Bioinformatics. 2014;30(14):2068-9. https://doi.org/10.1093/bioinformatics/btu153.

50. Page AJ, Cummins CA, Hunt M, Wong VK, Reuter S, Holden MTG, et al. Roary: rapid large-scale prokaryote pan genome analysis. Bioinformatics. 2015;31(22):3691-3. https://doi.org/10.1093/bioinformatics/btv421.

51. Katoh K, Standley DM. MAFFT multiple sequence alignment software version 7: improvements in performance and usability. Mol Biol Evol. 2013; 30(4):772-80. https://doi.org/10.1093/molbev/mst010.

52. Stamatakis A. RAxML version 8: a tool for phylogenetic analysis and postanalysis of large phylogenies. Bioinformatics. 2014;30(9):1312-3. https://doi. org/10.1093/bioinformatics/btu033.

53. Tavaré S. Some probabilistic and statistical problems in the analysis of DNA sequences. In: American mathematical society: lectures on mathematics in the life sciences: Amer Mathematical Society; 1986. p. 57-86. http://www.a mazon.ca/exec/obidos/redirect?tag=citeulike09-20\&path=ASIN/0821811673. Accessed 13 June 2013.

54. Letunic I, Bork P. Interactive tree of life (iTOL) v3: an online tool for the display and annotation of phylogenetic and other trees. Nucleic Acids Res. 2016:44(W1):W242-5. https://doi.org/10.1093/nar/gkw290. 
55. Blin K, Wolf T, Chevrette MG, Lu X, Schwalen CJ, Kautsar SA, et al. antiSMASH 4.0-improvements in chemistry prediction and gene cluster boundary identification. Nucleic Acids Res. 2017;45(W1):W36-41. https://doi. org/10.1093/nar/gkx319.

56. Bruen TC, Philippe H, Bryant D. A simple and robust statistical test for detecting the presence of recombination. Genetics. 2006;172(4):2665-81. https://doi.org/10.1534/genetics.105.048975.

57. Huson DH. SplitsTree: analyzing and visualizing evolutionary data. Bioinformatics. 1998;14(1):68-73. https://doi.org/10.1093/bioinformatics/14.1.68.

\section{Publisher's Note}

Springer Nature remains neutral with regard to jurisdictional claims in published maps and institutional affiliations.

Ready to submit your research? Choose BMC and benefit from:

- fast, convenient online submission

- thorough peer review by experienced researchers in your field

- rapid publication on acceptance

- support for research data, including large and complex data types

- gold Open Access which fosters wider collaboration and increased citations

- maximum visibility for your research: over $100 \mathrm{M}$ website views per year

At $\mathrm{BMC}$, research is always in progress.

Learn more biomedcentral.com/submissions 